TRANSACTIONS OF THE

AMERICAN MATHEMATICAL SOCIETY

Volume 360, Number 4, April 2008, Pages 1861-1877

S 0002-9947(07)04248-1

Article electronically published on November 19, 2007

\title{
THE CAUCHY PROBLEM AND INTEGRABILITY OF A MODIFIED EULER-POISSON EQUATION
}

\author{
FERIDE TIĞLAY
}

\begin{abstract}
We prove that the periodic initial value problem for a modified Euler-Poisson equation is well-posed for initial data in $H^{s}\left(\mathbb{T}^{m}\right)$ when $s>$ $m / 2+1$. We also study the analytic regularity of this problem and prove a Cauchy-Kowalevski type theorem. After presenting a formal derivation of the equation on the semidirect product space Diff $\ltimes C^{\infty}(\mathbb{T})$ as a Hamiltonian equation, we concentrate on one space dimension $(m=1)$ and show that the equation is bihamiltonian.
\end{abstract}

In this paper we study the periodic Cauchy problem for the modified EulerPoisson equation $(\mathrm{mEP})$

$$
\begin{aligned}
& \partial_{t} n+\operatorname{div}(n v)=0, \\
& \partial_{t} v+(v \cdot \nabla) v+\operatorname{grad} \Lambda^{-2} n=0, x \in \mathbb{T}^{m}, t \in \mathbb{R},
\end{aligned}
$$

as well as its Hamiltonian structure and integrability.

The equation $(\mathrm{mEP})$ is related to the Euler-Poisson equation

$$
\begin{aligned}
& \partial_{t} n+\operatorname{div}(n v)=0, \\
& \partial_{t} v+(v \cdot \nabla) v+\operatorname{grad} \phi=0, \\
& \Delta \phi-e^{\phi}+n=0, x \in \mathbb{T}^{m}, t \in \mathbb{R},
\end{aligned}
$$

which describes the fluctuations in the ion density of a two-component plasma of positively charged ions and negatively charged electrons (therefore it is also called ion acoustic plasma equation [LiSat]).

Linearizing the operator $N(\phi)=e^{\phi}-\Delta \phi$ at $\phi=0$ in the Euler-Poisson equation (1), we obtain the local form of the modified Euler-Poisson equation (mEP):

$$
\begin{aligned}
& \partial_{t} n+\operatorname{div}(n v)=0, \\
& \partial_{t} v+(v \cdot \nabla) v+\operatorname{grad} \phi=0, \\
& \Delta \phi-\phi+n=0, x \in \mathbb{T}^{m}, t \in \mathbb{R} .
\end{aligned}
$$

Equation (2), like the Euler-Poisson equation (1), admits an approximation which preserves dispersion and leads to KdV (see Remark 1). Inverting the operator $\Lambda:=I-\Delta$, we write the system in (2) in the nonlocal form (mEP).

Besides its relation to the Euler-Poisson equation (1), the modified Euler-Poisson equation is also remarkable for its bihamiltonian structure in one space dimension that we describe here.

Received by the editors October 15, 2004 and, in revised form, June 9, 2005 and October 13, 2005 .

2000 Mathematics Subject Classification. Primary 35Q53, 35Q05, 35A10, 37K65.

(C)2007 American Mathematical Society Reverts to public domain 28 years from publication 
Many different techniques have been developed based on Picard's contraction theorem on Banach spaces in the study of nonlinear partial differential equations. One approach originated in an observation of V. Arnold [Arn] that the initial value problem for the classical Euler equations of a perfect fluid can be stated as a problem of finding geodesics on the group of volume preserving diffeomorphisms. Subsequently, this observation was used by D.G. Ebin and J. Marsden in [EMa] who developed the necessary functional analytic tools and established sharp local well-posedness results for the Euler equations in a class of Sobolev spaces.

The first section of this work is devoted to developing an appropriate analytic framework for the modified Euler-Poisson equation (mEP) using a similar approach and proving the following theorem.

Theorem 1. For $s>m / 2+1$, given any initial data $\left(n_{0}, v_{0}\right) \in H^{s-1}\left(\mathbb{T}^{m}, \mathbb{R}\right) \times$ $H^{s}\left(\mathbb{T}^{m}, \mathbb{R}^{m}\right)$, there exists a $T>0$ and a unique solution $(n, v)$ to the Cauchy problem for the modified Euler-Poisson equation ( $m E P$ ) such that

$$
v \in C\left([0, T), H^{s}\left(\mathbb{T}^{m}, \mathbb{R}^{m}\right)\right) \cap C^{1}\left([0, T), H^{s-1}\left(\mathbb{T}^{m}, \mathbb{R}^{m}\right)\right)
$$

and

$$
n \in C\left([0, T), H^{s-1}\left(\mathbb{T}^{m}, \mathbb{R}\right)\right) \cap C^{1}\left([0, T), H^{s-2}\left(\mathbb{T}^{m}, \mathbb{R}\right)\right),
$$

and the solution $(n, v)$ depends continuously on the initial data $\left(n_{0}, v_{0}\right)$.

Another powerful tool in the study of partial differential equations is the CauchyKowalevski theorem. An abstract version of this theorem was developed by L.V. Ovsjannikov [Ovs1, Ovs2], F. Treves [Tre], L. Nirenberg [Nir], T. Nishida [Nis] and M.S. Baouendi and C. Goulaouic [BG] among others and subsequently applied to the Euler and Navier-Stokes equations.

The study of analytic regularity of solutions to the Camassa-Holm equation by A. Himonas and G. Misiołek [HM1], [HM3] using this abstract theorem led us to investigate the analytic regularity for $(\mathrm{mEP})$. In Section 2, we prove the existence and uniqueness of local analytic solutions to the Cauchy problem for the equation $(\mathrm{mEP})$.

Theorem 2. If the initial data $\left(n_{0}, v_{0}\right)$ is analytic on $\mathbb{T}^{m} \times \mathbb{T}^{m}$, then there exists an $\varepsilon>0$ and a unique solution $(n, v)$ of the Cauchy problem for the equation (mEP) that is analytic both in $x$ and $t$ on $\mathbb{T}^{m} \times \mathbb{T}^{m}$ for all $t$ in $(-\varepsilon, \varepsilon)$.

This result can be viewed as a Cauchy-Kowalevski type result for the equation $(\mathrm{mEP})$. Even though the equation (mEP) admits an approximation by the Korteweg-De Vries equation, the analytic regularity results for the two equations are quite different. In contrast with the Korteweg-De Vries equation whose solutions are analytic in the space variable for all time but not analytic in the time variable (see [Tru], [KaM]), the solutions to the modified Euler-Poisson equation are analytic in both space and time variables.

In the third section we derive the equation $(\mathrm{mEP})$ as a Hamiltonian equation on the semidirect product space $\operatorname{Diff}\left(\mathbb{T}^{m}\right) \ltimes C^{\infty}\left(\mathbb{T}^{m}\right)$ following the treatment of V. Arnold and B. Khesin in $[\mathrm{AK}]$ and J. Marsden, T. Ratiu and A. Weinstein in [MRW] of the Hamiltonian formalism related to fluid and gas dynamics. Then we concentrate on the one space dimension $m=1$ and prove the following theorem. 
Theorem 3. For $m=1$ the modified Euler-Poisson equation ( $m E P)$ is bihamiltonian with the pair of Hamiltonian functionals

and

$$
H_{1}=\int \frac{1}{2}\left(v^{2} n+\left(\Lambda^{-2} \partial_{x} n\right)^{2}+\left(\Lambda^{-2} n\right)^{2}\right) d x
$$

$$
H_{2}=\int n v d x
$$

In the proof we use prolongations to check the compatibility of the induced Poisson brackets by these Hamiltonian structures. In particular the modified EulerPoisson equation ( $\mathrm{mEP}$ ) can be derived as a Hamiltonian equation on the semidirect product space of the Virasoro algebra with the smooth functions on the torus vir $\ltimes$ $C^{\infty}(\mathbb{T})$ along with a nonlocal hierarchy of equations called Hunter-Zheng equations (see [BDP] for the bihamiltonian structure of the Hunter-Zheng equations).

Remark 1. The Korteweg-de Vries equation (KdV) can be derived as an approximation to the Euler-Poisson equation by a perturbation analysis (see [Sat]). Using this approach it is straightforward to obtain an approximation to the system of equations in (2) which preserves the dispersion and leads to $\mathrm{KdV}$.

\section{Local Well-Posedness in Sobolev SpaCeS}

In this section we study the Cauchy problem for the modified Euler-Poisson equation (mEP) where $n=n(t, x): \mathbb{R} \times \mathbb{T}^{m} \rightarrow \mathbb{R}$ and $v=v(t, x): \mathbb{R} \times \mathbb{T}^{m} \rightarrow \mathbb{R}^{m}$ and $\Lambda^{-2}=(I-\Delta)^{-1}$ is the Bessel potential.

In order to prove Theorem 1 we use the method of first restating the problem as an initial value problem for an ordinary differential equation on the group of diffeomorphisms of Sobolev class $H^{s}$ and then applying the existence theorem for vector fields on Banach manifolds.

Proposition 1. For $s>m / 2+1$, a pair $(n, v) \in H^{s-1}\left(\mathbb{T}^{m}, \mathbb{R}\right) \times H^{s}\left(\mathbb{T}^{m}, \mathbb{R}^{m}\right)$ is a solution to the Cauchy problem for $(m E P)$ with initial data $\left(n_{0}, v_{0}\right)$ if and only if $v=\eta \circ \gamma^{-1}$ and $n=\zeta \circ \gamma^{-1}$, where $(\gamma, \zeta, \eta)$ is a solution to

$$
\begin{aligned}
& \partial_{t} \zeta=-\left(\left(\zeta \circ \gamma^{-1}\right) \operatorname{div}\left(\eta \circ \gamma^{-1}\right)\right) \circ \gamma, \\
& \partial_{t} \eta=-\left(\operatorname{grad}\left(\Lambda^{-2}\left(\zeta \circ \gamma^{-1}\right)\right)\right) \circ \gamma, \\
& \partial_{t} \gamma=\eta,
\end{aligned}
$$

with initial conditions $\zeta(0, x)=n_{0}(x), \eta(0, x)=v_{0}(x), \gamma(0, x)=i d_{x}$.

Therefore the Cauchy problem for $(\mathrm{mEP})$ can be reformulated as an initial value problem for the ordinary differential equation

$$
\frac{d}{d t}(\gamma, \zeta, \eta)=(\eta, F(\gamma, \eta, \zeta), G(\gamma, \zeta))
$$

where

$$
\begin{gathered}
G(\gamma, \zeta)=-\left(\operatorname{grad} \Lambda^{-2}\left(\zeta \circ \gamma^{-1}\right)\right) \circ \gamma, \\
\left.F(\gamma, \zeta, \eta)=-\left(\left(\zeta \circ \gamma^{-1}\right) \operatorname{div}\left(\eta \circ \gamma^{-1}\right)\right) \circ \gamma\right)
\end{gathered}
$$

with initial data $\left(\gamma_{0}, \eta_{0}, \zeta_{0}\right)=\left(i d_{x}, v_{0}(x), n_{0}(x)\right)$.

In the proof of Theorem 1 we repeatedly use three standard results on Sobolev spaces: The Schauder ring property, the Sobolev imbedding theorem (that we refer 
to as the Sobolev lemma) and the composition lemma (see, for example, $[\mathrm{S}]$ and $[\mathrm{Ad}])$.

Proof of Theorem 1. If the map

$$
\begin{aligned}
\mathcal{D}^{s}\left(\mathbb{T}^{m}\right) \times H^{s-1}\left(\mathbb{T}^{m}, \mathbb{R}\right) \times H^{s}\left(\mathbb{T}^{m}, \mathbb{R}^{m}\right) \rightarrow & H^{s}\left(\mathbb{T}^{m}, \mathbb{R}^{m}\right) \times H^{s-1}\left(\mathbb{T}^{m}, \mathbb{R}\right), \\
& \times H^{s}\left(\mathbb{T}^{m}, \mathbb{R}^{m}\right) \\
(\gamma, \zeta, \eta) \mapsto \quad & (\eta, F, G)
\end{aligned}
$$

is locally Lipschitz, then by the fundamental theorem for ordinary differential equations on Banach spaces [Di] there is a unique solution

$$
(\gamma, \eta, \zeta) \in \mathcal{D}^{s}\left(\mathbb{T}^{m}\right) \times H^{s}\left(\mathbb{T}^{m}, \mathbb{R}^{m}\right) \times H^{s-1}\left(\mathbb{T}^{m}, \mathbb{R}\right)
$$

to the problem (4) for $s>m / 2+1$ with initial data

$$
\zeta(0, x)=n_{0}(x), \eta(0, x)=v_{0}(x), \gamma(0, x)=x .
$$

Note that the dependence of the solution of problem (4) on initial data is smooth. However the map $\gamma \mapsto \gamma^{-1}$ on $\mathcal{D}^{s}$ is continuous but not on $C^{1}$. Therefore we only have continuous dependence on initial data of the solution to the Cauchy problem for $(\mathrm{mEP})$.

By Proposition 1 the proof of Theorem 1 is reduced to showing that the maps

$$
\begin{aligned}
& \gamma \mapsto F(\gamma, \eta, \zeta) \in L\left(H^{s}\left(\mathbb{T}^{m}, \mathbb{R}^{m}\right), H^{s-1}\left(\mathbb{T}^{m}, \mathbb{R}\right)\right), \\
& \zeta \mapsto F(\gamma, \eta, \zeta) \in L\left(H^{s-1}\left(\mathbb{T}^{m}, \mathbb{R}\right), H^{s-1}\left(\mathbb{T}^{m}, \mathbb{R}\right)\right), \\
& \eta \mapsto F(\gamma, \eta, \zeta) \in L\left(H^{s}\left(\mathbb{T}^{m}, \mathbb{R}^{m}\right), H^{s-1}\left(\mathbb{T}^{m}, \mathbb{R}\right)\right), \\
& \gamma \mapsto G(\gamma, \zeta) \in L\left(H^{s}\left(\mathbb{T}^{m}, \mathbb{R}^{m}\right), H^{s}\left(\mathbb{T}^{m}, \mathbb{R}^{m}\right)\right), \\
& \zeta \mapsto G(\gamma, \zeta) \in L\left(H^{s-1}\left(\mathbb{T}^{m}, \mathbb{R}\right), H^{s}\left(\mathbb{T}^{m}, \mathbb{R}^{m}\right)\right)
\end{aligned}
$$

are locally Lipschitz in $\gamma, \eta$ and $\zeta$ (uniformly with respect to the remaining variables).

In the following estimates, the subscripts $\gamma$ and $\zeta$ of a constant indicate the dependence of the constant on $\|\gamma\|_{H^{s}}$ and $\|\zeta\|_{H^{s-1}}$ respectively.

$\gamma \mapsto G(\gamma, \zeta)$ is locally Lipschitz. Let $\gamma_{1}, \gamma_{2} \in \mathcal{D}^{s}\left(\mathbb{T}^{m}\right)$ and $\bar{\zeta} \in H^{s-1}\left(\mathbb{T}^{m}, \mathbb{R}\right)$. By the composition lemma,

$$
\begin{aligned}
& \left\|\operatorname{grad} \Lambda^{-2}\left(\bar{\zeta} \circ \gamma_{1}^{-1}\right) \circ \gamma_{1}-\operatorname{grad} \Lambda^{-2}\left(\bar{\zeta} \circ \gamma_{2}^{-1}\right) \circ \gamma_{2}\right\|_{H^{s}} \\
& \leq C_{\gamma_{2}}\left\|\operatorname{grad} \Lambda^{-2}\left(\bar{\zeta} \circ \gamma_{1}^{-1}\right) \circ \gamma-\operatorname{grad} \Lambda^{-2}\left(\bar{\zeta} \circ \gamma_{2}^{-1}\right)\right\|_{H^{s}},
\end{aligned}
$$

where $\gamma=\gamma_{1} \circ \gamma_{2}^{-1}$. Let $\zeta=\bar{\zeta} \circ \gamma_{2}^{-1}$. Then it is enough to show that the following estimate holds:

$$
\left\|\operatorname{grad} \Lambda^{-2}\left(\zeta \circ \gamma^{-1}\right) \circ \gamma-\operatorname{grad} \Lambda^{-2} \zeta\right\|_{H^{s}} \leq C_{\gamma, \zeta}\left\|\gamma-i d_{x}\right\|_{H^{s}} .
$$

Next we show that (6) holds for $s>m / 2+1$. The left side of the inequality (6) has the following form:

$$
\begin{aligned}
& \left\|\operatorname{grad} \Lambda^{-2}\left(\zeta \circ \gamma^{-1}\right) \circ \gamma-\operatorname{grad} \Lambda^{-2} \zeta\right\|_{H^{s}} \\
& \simeq\left\|\operatorname{grad} \Lambda^{-2}\left(\zeta \circ \gamma^{-1}\right) \circ \gamma-\operatorname{grad} \Lambda^{-2} \zeta\right\|_{L^{2}} \\
& +\left\|\operatorname{div}\left(\operatorname{grad} \Lambda^{-2}\left(\zeta \circ \gamma^{-1}\right) \circ \gamma-\operatorname{grad} \Lambda^{-2} \zeta\right)\right\|_{H^{s-1}}
\end{aligned}
$$


We first estimate the $L^{2}$ term in (7). For any $r>m / 2+\sigma$ we have the Sobolev imbedding into a Hölder space

$$
H^{r}\left(\mathbb{T}^{m}\right) \hookrightarrow C^{\sigma}\left(\mathbb{T}^{m}\right)
$$

with a bound

$$
|u(x)-u(y)| \leq C\|u\|_{H^{r}}|x-y|^{\sigma},
$$

for any $x, y \in \mathbb{T}$. Therefore using the composition lemma and applying (9) with $r=s-1$ and $\sigma=(s-1-m / 2) / 2$ we find

$$
\begin{aligned}
& \quad\left\|\operatorname{grad} \Lambda^{-2}\left(\zeta \circ \gamma^{-1}\right) \circ \gamma-\operatorname{grad} \Lambda^{-2} \zeta\right\|_{L^{2}} \\
& \leq\left\|\operatorname{grad} \Lambda^{-2}\left(\zeta \circ \gamma^{-1}\right) \circ \gamma-\operatorname{grad} \Lambda^{-2}\left(\zeta \circ \gamma^{-1}\right)\right\|_{L^{2}} \\
& \quad+\left\|\operatorname{grad} \Lambda^{-2}\left(\zeta \circ \gamma^{-1}\right)-\operatorname{grad} \Lambda^{-2} \zeta\right\|_{L^{2}} \\
& \leq C_{\gamma}\|\zeta\|_{H^{s-1}}\left(\left\|\gamma-i d_{x}\right\|_{H^{s}}^{\sigma+1}+\left\|\gamma^{-1}-i d_{x}\right\|_{H^{s}}^{\sigma}\right) .
\end{aligned}
$$

Adding and subtracting the appropriate terms we estimate the $H^{s-1}$ term in (8) by the sum

$$
\begin{aligned}
\leq & \left\|\sum_{i=1}^{n} \sum_{j=1}^{n} \partial_{j} \partial_{i} \Lambda^{-2}\left(\zeta \circ \gamma^{-1}\right) \circ \gamma\left(\partial_{i} \gamma_{j}-\delta_{i}^{j}\right)\right\|_{H^{s-1}} \\
& +\left\|\Delta \Lambda^{-2}\left(\zeta \circ \gamma^{-1}\right) \circ \gamma-\Delta \Lambda^{-2} \zeta\right\|_{H^{s-1}} .
\end{aligned}
$$

Using Schauder ring property and composition lemma the first summand in (10) is bounded by

$$
\leq\|\zeta\|_{H^{s-1}}\left\|\gamma-i d_{x}\right\|_{H^{s}} .
$$

In order to estimate the second summand (11) we add and subtract the terms $\Lambda^{-2}\left(\zeta \circ \gamma^{-1}\right) \circ \gamma$ and $\Lambda^{-2} \zeta$. After cancellations we obtain

$$
\begin{aligned}
& =\left\|\Lambda^{-2}\left(\zeta \circ \gamma^{-1}\right) \circ \gamma-\Lambda^{-2} \zeta\right\|_{H^{s-1}} \\
& \leq\left\|\Lambda^{-2}\left(\zeta \circ \gamma^{-1}\right) \circ \gamma-\Lambda^{-2}\left(\zeta \circ \gamma^{-1}\right)\right\|_{H^{s-1}} \\
& +\left\|\Lambda^{-2}\left(\zeta \circ \gamma^{-1}\right)-\Lambda^{-2} \zeta\right\|_{H^{s-1}} .
\end{aligned}
$$

Let $u$ be $\Lambda^{-2}\left(\zeta \circ \gamma^{-1}\right)$. Then we have

$$
\begin{aligned}
\left\|\Lambda^{-2}\left(\zeta \circ \gamma^{-1}\right) \circ \gamma-\Lambda^{-2}\left(\zeta \circ \gamma^{-1}\right)\right\|_{H^{s-1}} & =\left\|\int_{x}^{\gamma(x)} D u(y) d y\right\|_{H^{s-1}} \\
& \leq\|u\|_{C^{1}}\left\|\gamma-i d_{x}\right\|_{H^{s-1}}
\end{aligned}
$$

Using the Sobolev lemma with the composition lemma we obtain the estimate

$$
\begin{aligned}
& \leq\|u\|_{H^{s}}\left\|\gamma-i d_{x}\right\|_{H^{s-1}} \\
& \leq C_{\gamma}\|\zeta\|_{H^{s-1}}\left\|\gamma-i d_{x}\right\|_{H^{s}}
\end{aligned}
$$

for (12).

For $s>m / 2+2$ the term (13) can easily be estimated like (12). For $m / 2+1<$ $s \leq m / 2+2$ we first observe that the estimate

$$
\left\|\Lambda^{-2}\left(\zeta \circ \gamma^{-1}\right)-\Lambda^{-2} \zeta\right\|_{H^{s-1}} \leq\left\|\zeta \circ \gamma^{-1}-\zeta\right\|_{L^{2}}
$$


holds for $s-3 \leq 0$, and then using (9) as before, we obtain the following estimate for $(13)$ :

$$
\leq C_{\gamma}\|\zeta\|_{H^{s-1}}\left\|\gamma^{-1}-i d_{x}\right\|_{H^{s}}^{\sigma}
$$

where $\sigma$ is equal to $(s-1-m / 2) / 2>0$. However the assumption $s-3 \leq 0$ does not follow from $s \leq m / 2+2$ if $m \geq 3$. Nevertheless one can use the following inductive argument until $s-(2 k+1) \leq 0$ (it ends in finitely many steps since $s \leq m / 2+2$ ).

If $s-3>0$ we split (13) as in (7)-(8). The $L^{2}$ part can be estimated as for $s-3 \leq 0$. The $H^{s-2}$ part

$$
\begin{aligned}
& \left\|D\left(\Lambda^{-2}\left(\zeta \circ \gamma^{-1}\right) \circ \gamma-\Lambda^{-2} \zeta\right)\right\|_{H^{s-2}} \\
& =\left\|\operatorname{grad} \Lambda^{-2}\left(\zeta \circ \gamma^{-1}\right) \circ \gamma \cdot D \gamma-\operatorname{grad} \Lambda^{-2} \zeta\right\|_{H^{s-2}}
\end{aligned}
$$

is bounded by

$$
\begin{aligned}
& \leq\left\|\operatorname{grad} \Lambda^{-2}\left(\zeta \circ \gamma^{-1}\right) \circ \gamma \cdot(D \gamma-1)\right\|_{H^{s-2}} \\
& +\left\|\operatorname{grad} \Lambda^{-2}\left(\zeta \circ \gamma^{-1}\right) \circ \gamma-\operatorname{grad} \Lambda^{-2} \zeta\right\|_{H^{s-2}} .
\end{aligned}
$$

Here the first summand (18) is estimated using the Schauder ring property with the composition lemma

$$
\left\|\operatorname{grad} \Lambda^{-2}\left(\zeta \circ \gamma^{-1}\right) \circ \gamma \cdot(D \gamma-1)\right\|_{H^{s-2}} \leq C_{\gamma}\|\zeta\|_{H^{s-1}}\left\|\gamma-i d_{x}\right\|_{H^{s}} .
$$

For the second summand (19) we use the steps (6)-(14) to reduce it to estimating

$$
\left\|\Lambda^{-2}\left(\zeta \circ \gamma^{-1}\right)-\Lambda^{-2} \zeta\right\|_{H^{s-3}} .
$$

If $s-5 \leq 0$ we proceed as in (15)-(16). Otherwise we repeat the steps (17)-(20).

$\gamma \mapsto F(\gamma, \zeta, \eta)$ is locally Lipschitz. Let $\gamma_{1}, \gamma_{2} \in H^{s}\left(\mathbb{T}^{m}, \mathbb{R}^{m}\right)$ and

$$
\zeta \in H^{s-1}\left(\mathbb{T}^{m}, \mathbb{R}\right), \eta \in H^{s}\left(\mathbb{T}^{m}, \mathbb{R}^{m}\right) .
$$

Then by the Schauder ring property we have

$$
\begin{aligned}
& \left\|\zeta\left(\operatorname{div}\left(\eta \circ \gamma_{1}^{-1}\right) \circ \gamma_{1}\right)-\zeta\left(\operatorname{div}\left(\eta \circ \gamma_{2}^{-1}\right) \circ \gamma_{2}\right)\right\|_{H^{s-1}} \\
& \leq\|\zeta\|_{H^{s-1}}\left\|\sum_{j, m} \partial_{m} \eta_{j} \partial_{j}\left(\gamma_{1}^{-1}\right)_{m} \circ \gamma_{1}-\partial_{m} \eta_{j} \partial_{j}\left(\gamma_{2}^{-1}\right)_{m} \circ \gamma_{2}\right\|_{H^{s-1}} \\
& \leq C_{\gamma_{1}, \gamma_{2}, \zeta} \sum_{j, m}\left\|\partial_{m} \eta_{j}\right\|_{H^{s-1}}\left\|\partial_{j}\left(\gamma_{1}^{-1}\right)_{m}-\partial_{j}\left(\gamma_{2}^{-1}\right)_{m}\right\|_{H^{s-1}} .
\end{aligned}
$$

Using the Schauder ring property one more time we bound this term by

$$
\leq C_{\gamma_{1}, \gamma_{2}, \zeta, \eta}\|\eta\|_{H^{s}}\left\|\gamma_{1}-\gamma_{2}\right\|_{H^{s}},
$$

and therefore $\gamma \mapsto F(\gamma, \zeta, \eta)$ is locally Lipschitz.

It is straightforward to show that the second, third and fifth maps in (5) are uniformly Lipschitz using properties of Sobolev spaces. This completes the proof of Theorem 1.

Next we observe that the Cauchy problems for the equation (mEP) and the Euler equations of an incompressible fluid are not only similar for low regularity (Sobolev class) data but also for high regularity (analytic) data. 


\section{Analytic Regularity}

In this section we give a proof of Theorem 2 that states the analytic regularity (i.e., existence and uniqueness of analytic solutions for analytic initial data) of the Cauchy problem for (mEP).

Our approach is motivated by the work of M.S. Baouendi and C. Goulaouic $[\mathrm{BG}]$ who studied analytic regularity of the Cauchy problem for Euler equations of incompressible fluids.

The proof of Theorem 2 relies on a contraction argument in a decreasing scale of Banach spaces $X_{s}$ (i.e., if $s^{\prime}<s$ implies $X_{s} \subset X_{s^{\prime}}$ and $\left.\||\cdot|\|\right|_{s^{\prime}} \leq\|\| \cdot\|\|_{s}$ ).

For $s>0$, let the spaces $E_{s}$ be defined as

$$
E_{s}=\left\{u \in C^{\infty}\left(\mathbb{T}^{m}\right): \int_{\mathbb{T}^{m}} u d x=0 \text { and }\||u|\|_{s}=\sup _{|k| \geq 0} \frac{\left\|\partial_{x}^{k} u\right\|_{H^{\sigma}} s^{|k|}}{k ! /(|k|+1)^{2}}<\infty\right\},
$$

where $\sigma$ is any integer such that $\sigma>1+m / 2$ and let $X_{s}$ be given by the Cartesian product $E_{s} \times E_{s}$. The norm \|\|$\cdot\|\|_{X_{s}}$ can be chosen to be any of the standard product norms on $E_{s} \times E_{s}$. The following lemma states the ring property for the spaces $E_{s}$.

Lemma 1. Let $0<s<1$. There is a constant $c>0$ which is independent of $s$ such that we have

$$
\|u v\|_{s} \leq c\left|\|u \mid\|\left\|_{s}\right\| v \|_{s}\right.
$$

for any $u, v \in E_{s}$.

We omit the proof of Lemma 1 (see [HM1] for the case $m=1$ ). First we rewrite the equation $(\mathrm{mEP})$ in a more convenient form. Let $n$ and $v$ be denoted by $u_{1}$ and $u_{2}$ respectively. Then we can write the equation $(\mathrm{mEP})$ in terms of $\left(u_{1}, u_{2}\right)$ as

$$
\begin{aligned}
& \partial_{t} u_{1}=F_{1}\left(u_{1}, u_{2}\right):=\mathcal{P}_{2}\left(u_{1} u_{2}\right), \\
& \partial_{t} u_{2}=F_{2}\left(u_{1}, u_{2}\right):=\mathcal{P}_{4}\left(u_{2}\right) u_{2}+\mathcal{P}_{1} \mathcal{P}_{3} u_{1}
\end{aligned}
$$

where

$$
\begin{array}{ll}
\mathcal{P}_{1}(n):=-\operatorname{grad}(n), & \mathcal{P}_{3}(n):=\Lambda^{-2} n \\
\mathcal{P}_{2}(v):=-\operatorname{div}(v), & \mathcal{P}_{4}(u) v:=-\left(\nabla_{v} u\right)=-(D u) v .
\end{array}
$$

The following lemmas give the suitable bounds on these operators to prove Theorem 2 .

Lemma 2. For $0<s^{\prime}<s<1$, we have

$$
\begin{aligned}
& \|\| \mathcal{P}_{1} n\|\|_{s^{\prime}} \leq \frac{C}{s-s^{\prime}} \mid\|n\| \|_{s}, \\
& \left\|\mathcal{P}_{2} v\right\|\left\|_{s^{\prime}} \leq \frac{c}{s-s^{\prime}}\right\| v \|_{s} .
\end{aligned}
$$

Proof. By the definition of $\||\cdot|\|_{s}$, we have

$$
\left\|\mid \mathcal{P}_{1} n\right\| \|_{s^{\prime}}=\sup _{|k| \geq 0} \frac{\left\|\partial^{k} \mathcal{P}_{1} n\right\|_{H^{\sigma} s^{\prime|k|}}}{k ! /(|k|+1)^{2}} .
$$

The $H^{\sigma}$ norm on the right hand side can be written in the local coordinates up to a constant as

$$
\left\|\partial^{k}(\operatorname{grad}(n))\right\|_{H^{\sigma}\left(\mathbb{T}^{m}, \mathbb{R}^{m}\right)} \simeq \sum_{j=1}^{m}\left\|\partial^{k} \partial_{j} n\right\|_{H^{\sigma}\left(\mathbb{T}^{m}, \mathbb{R}\right)}
$$


Then we have the estimates

$$
\begin{aligned}
\left\|\left|\mathcal{P}_{1} n \|\right|_{s^{\prime}}\right. & \leq c \sup _{|k| \geq 0} \sup _{|\beta|=1} \frac{\left\|\partial^{k+\beta} n\right\|_{H^{\sigma}} s^{|k|}}{k ! /(|k|+1)^{2}} \\
& =c \sup _{|k| \geq 0|\beta|=1} \sup _{\mid\left\|\partial^{k+\beta} n\right\|_{H \sigma} s^{|k|+1}} \frac{(k+\beta) !}{(k+\beta) ! /(|k|+2)^{2}} \frac{s^{\prime|k|}}{(|k|+2)^{2}} \frac{(|k|+1)^{2}}{s^{|k|+1}} .
\end{aligned}
$$

Note that

$$
\sup _{|k| \geq 0} \sup _{|\beta|=1} \frac{\left\|\partial^{k+\beta} n\right\|_{H^{\sigma}} s^{|k|+1}}{(k+\beta) ! /(|k|+2)^{2}} \leq\|\| n\|\|_{s}
$$

Therefore we have

$$
\left\|\left|\mathcal{P}_{1} n\right|\right\|_{s^{\prime}} \leq m\left|\left\|n|\||_{s} \sup _{|k| \geq 0|\beta|=1} \sup _{|\beta|} \frac{s^{\prime|k|}}{s^{|k|+1}} \frac{(k+\beta) !}{k !}\left(\frac{|k|+1}{|k|+2}\right)^{2} .\right.\right.
$$

Note also that

$$
\sup _{|\beta|=1} \frac{(k+\beta) !}{k !}=\sup _{1 \leq i \leq m}\left(k_{i}+1\right) \leq|k|+1 .
$$

Then it follows from formula (24) that the inequality

$$
\sup _{|k| \geq 0} \sup _{|\beta|=1} \frac{s^{\prime|k|}}{s^{|k|+1}} \frac{(k+\beta) !}{k !}\left(\frac{|k|+1}{|k|+2}\right)^{2} \leq \frac{C}{s-s^{\prime}}
$$

holds. By (22) and (23) we obtain

$$
\left\|\left|\mathcal{P}_{1} n\left\|\left.\right|_{s^{\prime}} \leq \frac{C}{s-s^{\prime}} \mid\right\| n \|_{s} .\right.\right.
$$

The estimate for $\mathcal{P}_{2}$ follows similarly.

Lemma 3. For any $0<s<1$, the estimate

$$
\left\|\mathcal{P}_{3}(u) \mid\right\|_{s} \leq\|\| u \|_{s}
$$

holds if $u \in E_{s}$.

Lemma 4. For $0<s^{\prime}<s<1$, we have

$$
\left.\left\|\left|\mathcal{P}_{4}(u) v\right|\right\|\right|_{s^{\prime}} \leq \frac{c}{s-s^{\prime}}\|v\|\left\|_{s^{\prime}}\right\| u \mid\|\|_{s} .
$$

Proof. We write $\mathcal{P}_{4}(u) v$ in terms of the linear operator $D u$ as $\mathcal{P}_{4}(u) v=(D u) v$.

Then by Lemma 1 we have

$$
\begin{aligned}
\left\|\mathcal{P}_{4}(u) v\right\| \|_{s^{\prime}} & =\|\mid(D u) v\| \|_{s^{\prime}} \\
& =\left\|\mid \sum_{i, j=1}^{m} v_{i} \partial_{i} u_{j}\right\| \|_{s^{\prime}} \\
& \leq \sum_{i, j=1}^{m}\|\| v_{i}\left|\left\|s _ { s ^ { \prime } } \left|\left\|\partial_{i} u_{j} \mid\right\|_{s^{\prime}} .\right.\right.\right.
\end{aligned}
$$


Now we reduce the proof to the case that we handled in the proof of Lemma 2:

$$
\begin{aligned}
\left\|\mathcal{P}_{4}(u) v \mid\right\|_{s^{\prime}} & \leq c\left|\left\|v\left|\left\|_{s^{\prime}} \sum_{i, j=1}^{m}\right\| \partial_{i} u_{j} \|\right|_{s^{\prime}}\right.\right. \\
& \leq c\left|\|v \mid\|_{s^{\prime}} \sum_{j=1}^{m} \sup _{|k| \geq 0|\beta|=1} \frac{\left\|\partial^{k+\beta} u_{j}\right\|_{H^{\sigma}} s^{\prime|k|}}{k ! /(|k|+1)^{2}}\right. \\
& \leq c\left|\left\|v \left|\left\|_{s^{\prime}} \mid\right\| u\|\|_{s} \sup _{|k| \geq 0|\beta|=1} \sup _{|k|=1} \frac{s^{\prime|k|}}{s^{|k|+1}} \frac{(k+\beta) !}{k !}\left(\frac{|k|+1}{|k|+2}\right)^{2} .\right.\right.\right.
\end{aligned}
$$

Clearly, to finish the proof, it is enough to show that

$$
\frac{s^{\prime k}}{s^{k+1}}\left(\frac{k+1}{k+2}\right)^{2}(k+1) \leq \frac{1}{s-s^{\prime}} .
$$

Let $s^{\prime}=\lambda s, 0<\lambda<1$ and $f(\lambda)=(k+1)(1-\lambda) \lambda^{k}$. Then,

$$
\begin{aligned}
\frac{s^{\prime}}{s^{k+1}}\left(\frac{k+1}{k+2}\right)^{2}(k+1) & =\frac{\lambda^{k} s^{k}}{s^{k+1}}\left(\frac{k+1}{k+2}\right)^{2}(k+1) \\
& =\frac{1}{s(1-\lambda)} f(\lambda)\left(\frac{k+1}{k+2}\right)^{2} .
\end{aligned}
$$

For $k=0$ it is clear that $f(\lambda) \leq 1$. For $k \geq 1$ the function $f(\lambda)=(k+1)(1-\lambda) \lambda^{k}$ is continuous in the interval $0<\lambda<1$, and it has zeros at the endpoints of the interval $[0,1]$ and a maximum at $\lambda=\frac{k}{k+1}$ such that $f\left(\frac{k}{k+1}\right)=\left(\frac{k}{k+1}\right)^{k}<1$. Then we have

$$
\left(\lambda^{k}-\lambda^{k+1}\right)\left(\frac{k+1}{k+2}\right)^{2}(k+1) \leq 1 .
$$

Therefore

$$
\frac{\lambda^{k} s^{k}}{s^{k+1}}\left(\frac{k+1}{k+2}\right)^{2}(k+1) \leq \frac{1}{s(1-\lambda)},
$$

and the formula (24) holds. Using the formula (24) we obtain the desired estimate

$$
\left.\left\|\left|\mathcal{P}_{4}(u) v\right|\right\|\right|_{s^{\prime}} \leq \frac{c}{s-s^{\prime}}\|v\|\left\|_{s^{\prime}}\right\| u \mid \|_{s} .
$$

Now we are ready to prove Theorem 2 .

Proof of Theorem 2. We refer to the version of the abstract Cauchy-Kowalevski theorem in [Nis]. We only need to verify the first two conditions of this theorem since the map $F\left(u_{1}, u_{2}\right)$ does not depend on $t$ explicitly.

Clearly, $t \longmapsto F(t, u(t))=\left(F_{1}\left(u_{1}, u_{2}\right), F_{2}\left(u_{1}, u_{2}\right)\right)$ is holomorphic if $t \longmapsto u_{1}(t)$ and $t \longmapsto u_{2}(t)$ are both holomorphic. We only need to show that $F_{1}\left(u_{1}, u_{2}\right)$ and $F_{2}\left(u_{1}, u_{2}\right)$ are in $E_{s^{\prime}}$ if $u_{1}, u_{2} \in E_{s}$. We begin with estimates on $F_{1}$.

By Lemma 2 and Lemma 1, we have

$$
\left.\left\|\left|F_{1}\left(u_{1}, u_{2}\right)\right|\right\|\right|_{s^{\prime}}=\left\|| | \mathcal { P } _ { 2 } ( u _ { 1 } u _ { 2 } ) | \| | _ { s ^ { \prime } } \leq \frac { c } { s - s ^ { \prime } } \| | u _ { 1 } \left|\|\|_{s}\left\|\mid u_{2}\right\| \|_{s} .\right.\right.
$$

Similarly, for $F_{2}$, using Lemmas 2, 3 and 4 we have

$$
\left\|\left|F_{2}\left(u_{1}, u_{2}\right)\left\|\left.\right|_{s^{\prime}}=\right\|\right| \mathcal{P}_{4}\left(u_{2}\right) u_{2}+\mathcal{P}_{1} \mathcal{P}_{3} u_{1}||_{s^{\prime}} \leq \frac{c}{s-s^{\prime}}\left(\left.\left\|\left|u_{2}\right|\right\|\right|_{s} ^{2}+\left\|\left|u_{1} \|\right|_{s}\right) .\right.\right.
$$


We proceed to establish the second condition of the abstract Cauchy-Kowalevski theorem. We will show that for some $c$ independent of $t$,

$$
\left\|\left|F_{1}\left(u_{1}, u_{2}\right)-F_{1}\left(v_{1}, v_{2}\right)\right|\right\|_{s^{\prime}} \leq \frac{c}{s-s^{\prime}}\left\|u-v|\||_{X_{s}},\right.
$$

and similarly

$$
\left\||| F_{2}\left(u_{1}, u_{2}\right)-\left.F_{2}\left(v_{1}, v_{2}\right)||\right|_{s^{\prime}} \leq \frac{c}{s-s^{\prime}}\right\| \mid u-v\|\|_{X_{s}}
$$

where $u=\left(u_{1}, u_{2}\right)$ and $v=\left(v_{1}, v_{2}\right)$.

To obtain the first estimate above, after applying Lemma 2 , we add and subtract the term $u_{1} v_{2}$ and use Lemma 1 :

$$
\begin{aligned}
\left\|\left|F_{1}\left(u_{1}, u_{2}\right)-F_{1}\left(v_{1}, v_{2}\right) \|\right|_{s^{\prime}}\right. & =\|\left.\left|\mathcal{P}_{2}\left(u_{1} u_{2}-v_{1} v_{2}\right)\right|\right|_{s^{\prime}} \\
& \leq \frac{c}{s-s^{\prime}}\left(||\left|u_{1}\right|||_{s}||\left|u_{2}-v_{2}\right| \|_{s}+\left.||\left|u_{1}-v_{1}\right|||_{s}|| v_{2}||\right|_{s}\right) .
\end{aligned}
$$

Then, assuming that $\||u|\| \|_{s}<R$ and $\|\mid v\|_{s}<R$, we have

$$
\left\|\left|F_{1}\left(u_{1}, u_{2}\right)-F_{1}\left(v_{1}, v_{2}\right)\right|\right\|_{s^{\prime}} \leq \frac{c}{s-s^{\prime}}\|u-v\| \|_{X_{s}} .
$$

To estimate the $F_{2}$ component, we use Lemmas 2 and 3:

$$
\begin{array}{r}
\left\|\left|F_{2}\left(u_{1}, u_{2}\right)-F_{2}\left(v_{1}, v_{2}\right)\left\|\left.\right|_{s^{\prime}}=\right\|\right|\left(\mathcal{P}_{4} u_{2}\right) u_{2}-\left(\mathcal{P}_{4} v_{2}\right) v_{2}+\mathcal{P}_{1} \mathcal{P}_{3}\left(u_{1}-v_{1}\right)||_{s^{\prime}}\right. \\
\leq\left\|||\left(\mathcal{P}_{4} u_{2}\right) u_{2}-\left(\mathcal{P}_{4} v_{2}\right) v_{2}\left|\left\|_{s^{\prime}}+\frac{c}{s-s^{\prime}}||\left|u_{1}-v_{1}\right|\right\|\right|_{s}\right.
\end{array}
$$

Note that

$$
\mathcal{P}_{4}\left(u_{2}\right) u_{2}-\mathcal{P}_{4}\left(v_{2}\right) v_{2}=\mathcal{P}_{4}\left(u_{2}\right)\left(u_{2}-v_{2}\right)+\mathcal{P}_{4}\left(u_{2}-v_{2}\right) v_{2} .
$$

Using Lemma 4 and the above identity, (25) implies

$$
\begin{aligned}
& \|\left|F_{2}\left(u_{1}, u_{2}\right)-F_{2}\left(v_{1}, v_{2}\right)\right|||_{s^{\prime}} \leq \frac{c}{s-s^{\prime}}\left(||\left|u_{2}\right|\left|\|_{s}\right||| u_{2}-\left.v_{2}||\right|_{s^{\prime}}+\right. \\
& \left.+||\left|u_{2}-v_{2}\right|||_{s}||\left|v_{2}\right|||_{s^{\prime}}+\left.||\left|u_{1}-v_{1}\right|\right|_{s}\right) .
\end{aligned}
$$

Therefore the estimate

$$
\left\|\left|F_{2}\left(u_{1}, v_{1}\right)-F_{2}\left(u_{2}, v_{2}\right)\right|\right\|_{s^{\prime}} \leq \frac{c}{s-s^{\prime}}\|\mid u-v\| \|_{X_{s}}
$$

holds. This completes the proof of Theorem 2 .

\section{Bihamiltonian structure AND integrability}

A number of partial differential equations that describe fluid motion can be derived as equations for geodesics on various infinite dimensional Lie groups. For instance, the Euler equation for ideal incompressible fluid flow is the geodesic equation on the group of volume-preserving diffeomorphisms of a Riemannian manifold $M$ with a right invariant metric given by the $L^{2}$ inner product on the tangent space at the identity of the group [EMa]. Other examples are

- the Korteweg-de Vries equation and Camassa-Holm equation on the Bott-Virasoro group (see for example [OK] and [Mis3]),

- the ideal incompressible MHD (magnetohydrodynamics) on the semidirect product of volume preserving diffeomorphisms with the divergence free vector fields,

- the Hunter-Saxton equation on the homogeneous space of all diffeomorphisms of the unit circle modulo the rotations $[\mathrm{KM}]$, etc. 
In contrast with all the examples we gave above, the energy of the modified Euler-Poisson equation (mEP) is not a quadratic form, therefore it cannot be interpreted as a Riemannian metric. However, there still is a variational problem on the cotangent space of the configuration space of this equation. Here we derive the equation (mEP) from this variational problem. Note that all the computations that follow are formal.

Let $\mathfrak{g}$ be a Lie algebra with the bracket operation $[\cdot, \cdot]$ and $\mathfrak{g}^{*}$ be its dual given by the pairing

$$
\langle\cdot, \cdot\rangle: \mathfrak{g}^{*} \times \mathfrak{g} \rightarrow \mathbb{R}
$$

Then $\mathfrak{g}^{*}$ with the Lie-Poisson bracket defined by

for $F, G: \mathfrak{g}^{*} \rightarrow \mathbb{R}$ is a Poisson manifold.

$$
\{F, G\}(m)=\left\langle m,\left[\frac{\delta F}{\delta m}, \frac{\delta G}{\delta m}\right]\right\rangle
$$
by

$$
\dot{F}=\{F, H\} \text { for all } F \in C^{\infty} .
$$

Given a Lie-Poisson manifold, Hamilton's equations (26) can be written as

$$
\begin{aligned}
\dot{F}(m) & =\{F, H\}(m), \\
\left\langle\frac{\delta F}{\delta m}, \partial_{t} m\right\rangle & =-\left\langle m,\left[\frac{\delta H}{\delta m}, \frac{\delta F}{\delta m}\right]\right\rangle \\
& =-\left\langle a d_{\delta H / \delta m}^{*} m, \frac{\delta F}{\delta m}\right\rangle .
\end{aligned}
$$

Therefore

$$
\partial_{t} m=-a d_{\delta H / \delta m}^{*} m
$$

is an equivalent formulation of the Hamilton equation on a Lie-Poisson manifold.

Here we exploit the tools and techniques used to study the Hamiltonian formulation of the Euler equations for a compressible fluid [MRW] to show that the modified Euler-Poisson equation (mEP) can be derived as a Hamiltonian equation.

On the Cartesian product space $\operatorname{Diff}\left(\mathbb{T}^{m}\right) \times C^{\infty}\left(\mathbb{T}^{m}\right)$ of the group of diffeomorphisms of $\mathbb{T}^{m}$ and the vector space $C^{\infty}\left(\mathbb{T}^{m}\right)$ of all smooth functions on $\mathbb{T}^{m}$, the operation

$$
(\phi, a) \circ(\psi, b)=\left(\phi \circ \psi, a \circ \psi^{-1}+b\right),
$$

called the semidirect product, induces a Lie group structure. We denote this group by

$$
G=\operatorname{Diff}\left(\mathbb{T}^{m}\right) \ltimes C^{\infty}\left(\mathbb{T}^{m}\right)
$$

following the conventional notation for semidirect product spaces. The corresponding Lie algebra is the space $\mathfrak{g}=\operatorname{Vect}\left(\mathbb{T}^{m}\right) \ltimes C^{\infty}\left(\mathbb{T}^{m}\right)$ with the bracket

$$
[(v, a),(w, b)]=\left([v, w], \mathcal{L}_{v} b-\mathcal{L}_{w} a\right)
$$

where $v, w \in \operatorname{Vect}\left(\mathbb{T}^{m}\right)$ and $a, b \in C^{\infty}\left(\mathbb{T}^{m}\right)$. Here $[v, w]$ is the usual commutator of vector fields on $\mathbb{T}^{m}$ and $\mathcal{L}_{w} a$ is the Lie derivative of $a$ in the direction of $w$ and is given by $\mathcal{L}_{w} a=\left.\frac{d}{d s}\right|_{s=0}\left(a \circ \zeta^{s}\right)$ where $\zeta^{s}$ is any curve on Diff $\left(\mathbb{T}^{m}\right)$ such that $\left.\zeta^{s}\right|_{s=0}=i d$ and $\left.\frac{d}{d s}\right|_{s=0} \zeta^{s}=w$. Note that in this setting the composition of the diffeomorphisms is the group operation on Diff $\left(\mathbb{T}^{m}\right)$, and the composition of a smooth function with a diffeomorphism $a \circ \gamma^{-1}$ is the natural action of the 
diffeomorphism $\gamma$ on the function $a$. In general, the semidirect product structure on the Cartesian product of a Lie group and a vector space on which the group acts is defined using the group operation and the action of the group on the vector space (see $[\mathrm{AK}],[\mathrm{MRW}]$ ).

In this context, a Hamiltonian formulation of the modified Euler-Poisson equation $(\mathrm{mEP})$ can be stated as follows:

Theorem 4. The modified Euler-Poisson equation (mEP) is a Hamiltonian equation on $\mathfrak{g}^{*}$ with respect to the linear Lie-Poisson structure and the energy function

$$
H(M, n)=-\int_{\mathbb{T}^{m}}\left(\frac{1}{2 n}\langle M, M\rangle+\Phi(n)\right) d x
$$

where $M=n v \in \operatorname{Vect}\left(\mathbb{T}^{m}\right)$ and $\Phi^{\prime}(n)=\Lambda^{-2}(n)$.

Proof. We want to derive the equations for $v$ and $n$ from

$$
\partial_{t} m=-a d_{\delta H / \delta m}^{*} m
$$

where $m=(M, n)=(n v, n)$ and $(v, n) \in \mathfrak{g}^{*}$.

The variational derivative $\frac{\delta H}{\delta m}$ is given by $\left(\frac{\delta H}{\delta M}, \frac{\delta H_{1}}{\delta n}\right)$ with

$$
\begin{gathered}
\frac{\delta H_{1}}{\delta M}=-M / n=-v, \\
\frac{\delta H_{1}}{\delta n}=\frac{1}{2 n^{2}}\langle M, M\rangle-\Phi^{\prime}(n)=\frac{1}{2}\langle v, v\rangle-\Phi^{\prime}(n) .
\end{gathered}
$$

Evaluating equation $(29)$ on an arbitrary pair $(w, b) \in \mathfrak{g}$, we obtain

$$
\left\langle\partial_{t} m,(w, b)\right\rangle=-\left\langle a d_{\delta H_{1} / \delta m}^{*} m,(w, b)\right\rangle .
$$

Then by the definition of the coadjoint operator $a d^{*}$ and the bracket on $\mathfrak{g}$, we have

$$
\begin{aligned}
\left\langle\partial_{t} m,(w, b)\right\rangle & =-\left\langle(M, n),\left[\left(\frac{\delta H_{1}}{\delta M}, \frac{\delta H_{1}}{\delta n}\right),(w, b)\right]\right\rangle \\
& =\left\langle(M, n),\left(\left[w, \frac{\delta H_{1}}{\delta M}\right], \mathcal{L}_{w} \frac{\delta H_{1}}{\delta n}-\mathcal{L}_{\frac{\delta H_{1}}{\delta M}} b\right)\right\rangle .
\end{aligned}
$$

In what follows we identify the dual space $\mathfrak{g}^{*}$ with the algebra $\mathfrak{g}$ using the pairing $\langle\cdot, \cdot\rangle$ on $\mathfrak{g}^{*} \times \mathfrak{g}$ given by

$$
\langle(v, a),(w, b)\rangle=\int_{\mathbb{T}^{m}}\langle v, w\rangle d x+\int_{\mathbb{T}^{m}} a b d x .
$$

Then substituting $n v$ for $M$ and using (30) and (31), we obtain

$$
\begin{aligned}
\left\langle\partial_{t} m,(w, b)\right\rangle= & \int_{\mathbb{T}^{m}}\langle[w,-v], n v\rangle d x \\
& +\int_{\mathbb{T}^{m}} n\left(\mathcal{L}_{w}\left(\frac{1}{2}\langle v, v\rangle-\Phi^{\prime}(n)\right)-\mathcal{L}_{-v} b\right) d x .
\end{aligned}
$$

By the definition of the bracket $[\cdot, \cdot]$ on $\operatorname{Vect}\left(\mathbb{T}^{\mathrm{m}}\right)$, we have

$$
\begin{aligned}
\left\langle\partial_{t} m,(w, b)\right\rangle= & \int_{\mathbb{T}^{m}}\langle(v . \nabla) w, n v\rangle-\langle(w . \nabla) v, n v\rangle d x \\
& +\int_{\mathbb{T}^{m}} n\left(\mathcal{L}_{w}\left(\frac{1}{2}\langle v, v\rangle-\Phi^{\prime}(n)\right)-\mathcal{L}_{-v} b\right) d x .
\end{aligned}
$$


Furthermore, we can compute the Lie derivatives on the right hand side and write the above equality as follows:

$$
\begin{aligned}
\left\langle\partial_{t} m,(w, b)\right\rangle= & \int_{\mathbb{T}^{m}}\langle(v \cdot \nabla) w, n v\rangle-\langle(w \cdot \nabla) v, n v\rangle d x \\
& +\int_{\mathbb{T}^{m}}\left\langle n w, \operatorname{grad}\left(\frac{1}{2}\langle v, v\rangle-\Phi^{\prime}(n)\right)\right\rangle+\langle n v, \operatorname{grad}(b)\rangle d x .
\end{aligned}
$$

Using the identities

$$
\langle v, \operatorname{grad}\langle v, w\rangle\rangle=\langle w,(v \cdot \nabla) v\rangle+\langle v,(v \cdot \nabla) w\rangle
$$

and

$$
\left\langle w, \frac{1}{2} \operatorname{grad}\langle v, v\rangle\right\rangle=\langle v,(w \cdot \nabla) v\rangle
$$

we obtain

$$
\begin{aligned}
\left\langle\partial_{t} m,(w, b)\right\rangle= & \int_{\mathbb{T}^{m}}\langle\operatorname{grad}\langle v, w\rangle, n v\rangle-\langle(v . \nabla) v, n w\rangle \\
& \quad-\left\langle n w, \operatorname{grad}\left(\Phi^{\prime}(n)\right)\right\rangle+\langle n v, \operatorname{grad}(b)\rangle d x .
\end{aligned}
$$

Integrating by parts the first and the last summands on the right hand side, we obtain

$\left\langle\partial_{t} m,(w, b)\right\rangle=\int_{\mathbb{T}^{m}}-\operatorname{div}(n v)\langle v, w\rangle-\langle(v . \nabla) v, n w\rangle-\left\langle n w, \operatorname{grad}\left(\Phi^{\prime}(n)\right)\right\rangle-b \operatorname{div}(n v) d x$.

Note that

$$
\left\langle\partial_{t} m,(w, b)\right\rangle=\left\langle\left(\left(\partial_{t} n\right) v+n\left(\partial_{t} v\right), \partial_{t} n\right),(w, b)\right\rangle .
$$

Then by (32), we have

$$
\begin{gathered}
\partial_{t} n=-\operatorname{div}(n v) \\
\left(\partial_{t} n\right) v+n\left(\partial_{t} v\right)=-\operatorname{div}(n v) v-n(v \cdot \nabla) v-n \operatorname{grad}\left(\Phi^{\prime}(n)\right)
\end{gathered}
$$

which is equivalent to

$$
\begin{aligned}
& \partial_{t} n=-\operatorname{div}(n v), \\
& \partial_{t} v=-(v \cdot \nabla) v-\operatorname{grad}\left(\Phi^{\prime}(n)\right) .
\end{aligned}
$$

Note that for one space dimension $(m=1)$ the Hamiltonian $H_{1}$ in $(28)$ is given by

$$
H_{1}=\int \frac{1}{2}\left(v^{2} n+\left(\Lambda^{-2} \partial_{x} n\right)^{2}+\left(\Lambda^{-2} n\right)^{2}\right) d x
$$

in terms of $v$ and $n$. Then using the differential operator $\mathcal{D}_{1}$ that is defined as

$$
\mathcal{D}_{1}=\left(\begin{array}{rr}
0 & -\partial \\
-\partial & 0
\end{array}\right)
$$

one can rewrite the equation $(\mathrm{mEP})$ in the Hamiltonian form

$$
\partial_{t}\left(\begin{array}{c}
v \\
n
\end{array}\right)=\mathcal{D}_{1}\left(\begin{array}{l}
\delta H_{1} / \delta v \\
\delta H_{1} / \delta n
\end{array}\right) .
$$


The Poisson bracket induced by the matrix differential operator $\mathcal{D}_{1}$ is given by

$$
\begin{gathered}
\{F, H\}(v, n)=\left\langle(v, n),\left(\left[\left(\frac{\delta F}{\delta v}, \frac{\delta F}{\delta n}\right),\left(\frac{\delta H}{\delta v}, \frac{\delta H}{\delta n}\right)\right]\right)\right\rangle \\
=\int\left(\frac{\delta F}{\delta n} \partial_{x} \frac{\delta H}{\delta v}-\frac{\delta H}{\delta n} \partial_{x} \frac{\delta F}{\delta v}\right) d x .
\end{gathered}
$$

Another conserved quantity for $(\mathrm{mEP})$ is

$$
H_{2}=\int n v d x
$$

For one space dimension $(m=1)$ we can use $H_{2}$ to write $(\mathrm{mEP})$ in yet another form as

$$
\partial_{t}\left(\begin{array}{c}
v \\
n
\end{array}\right)=\mathcal{D}_{2}\left(\begin{array}{l}
\delta H_{2} / \delta v \\
\delta H_{2} / \delta n
\end{array}\right)
$$

where $\mathcal{D}_{2}$ is defined as

$$
\mathcal{D}_{2}=\left(\begin{array}{cc}
-\Lambda^{-2} \partial_{x} & -\partial_{x} v \\
\partial_{x} v & -(n \partial+\partial n)
\end{array}\right) .
$$

We prove Theorem 3 by showing that (34) and (36) are Hamiltonian forms of the modified Euler-Poisson equation $(\mathrm{mEP})$ and that the induced Poisson structures are compatible.

Proof of Theorem 3. The matrix differential operator $\mathcal{D}_{1}$ is skew-adjoint and does not depend on $v$ or $n$ nor any of their derivatives; therefore the bracket given by $\mathcal{D}_{1}$ satisfies the Jacobi identity; and hence is indeed a Poisson bracket.

We can easily check that $\mathcal{D}_{2}$ is skew-adjoint as well:

$$
\begin{gathered}
\int\left(\phi_{1}, \phi_{2}\right) \mathcal{D}_{2}\left(\theta_{1}, \theta_{2}\right) d x \\
=\int \phi_{1}\left(\Lambda^{-2} \partial_{x} \theta_{1}-\theta_{2} \partial_{x} v\right)+\phi_{2}\left(\rightarrow \partial_{x} v-n \partial_{x} \theta_{2}-\partial_{x}\left(n \theta_{2}\right)\right) d x \\
=-\int\left(\rightarrow \Lambda^{-2} \partial_{x} \phi_{1}-\phi_{2} \rightarrow \partial_{x} v+\phi_{1} \theta_{2} \partial_{x} v-\theta_{2} \partial_{x}\left(\phi_{2} n\right)-n \theta_{2} \partial_{x} \phi_{2}\right) d x \\
=\int\left(\theta_{1}, \theta_{2}\right) \mathcal{D}_{2}^{*}\left(\phi_{1}, \phi_{2}\right) d x .
\end{gathered}
$$

To verify the Jacobi identity for the bracket induced by $\mathcal{D}_{2}$ we adapt the notation of prolongations (see [Ol] for details). Let $\Theta_{2}$ be the functional bivector associated to $\mathcal{D}_{2}$ :

$$
\begin{gathered}
\Theta_{2}=\frac{1}{2} \int\left(\theta_{1}, \theta_{2}\right) \mathcal{D}_{2}\left(\theta_{1}, \theta_{2}\right) d x \\
=\frac{1}{2} \int\left\{\theta_{1} \wedge \Lambda^{-2} \partial_{x} \theta_{1}-\theta_{1} \wedge \theta_{2} \partial_{x} v\right. \\
\left.+\theta_{2} \wedge \theta_{1} \partial_{x} v-\theta_{2} \wedge n \partial_{x} \theta_{2}-\theta_{2} \wedge \partial_{x}\left(\theta_{2} n\right)\right\} d x \\
=\frac{1}{2} \int\left\{\theta_{1} \wedge \Lambda^{-2} \partial_{x} \theta_{1}-2\left(\partial_{x} v\right) \theta_{1} \wedge \theta_{2}-2 \theta_{2} \wedge n \partial_{x} \theta_{2}\right\} d x
\end{gathered}
$$


Then $\mathcal{D}_{2}$ is Hamiltonian since

$$
\begin{gathered}
\operatorname{pr} \mathbf{v}_{\mathcal{D}_{2} \theta}\left(\Theta_{2}\right)=-\frac{1}{2} \int\left\{2\left(\theta_{1} \partial_{x} v-n \partial_{x} \theta_{2}-\partial_{x}\left(\theta_{2} n\right)\right) \wedge \theta_{2} \wedge \partial_{x} \theta_{2}\right. \\
\left.+\partial_{x}\left(\Lambda^{-2} \partial_{x} \theta_{1}-\theta_{2} \partial_{x} v\right) \wedge \theta_{1} \wedge \theta_{2}\right\} d x \\
=-\int\left\{\Lambda^{-2} \partial_{x}^{2} \theta_{1} \wedge \theta_{1} \wedge \theta_{2}\right\} d x \\
=-\int\left\{\Lambda^{-2} \theta_{1} \wedge \theta_{1} \wedge \theta_{2}\right\} d x \\
=-\frac{1}{2} \int\left\{\partial_{x}^{2}\left(\Lambda^{-2} \theta_{1} \wedge \Lambda^{-2} \theta_{1}\right) \wedge \theta_{2}\right\} d x \\
=0
\end{gathered}
$$

These two Hamiltonian structures, (34) and (36), are compatible, i.e., the equation $(\mathrm{mEP})$ is bihamiltonian in one space dimension. To prove the compatibility it is enough to check that

$$
\operatorname{pr} \mathbf{v}_{\mathcal{D}_{1} \theta}\left(\Theta_{\mathcal{D}_{2}}\right)+\operatorname{pr} \mathbf{v}_{\mathcal{D}_{2} \theta}\left(\Theta_{\mathcal{D}_{1}}\right)=0
$$

holds, where $\Theta_{\mathcal{D}_{i}}$ denotes the corresponding bivector for $\mathcal{D}_{i}$. Both summands in (37) vanish:

$$
\begin{gathered}
\operatorname{pr} \mathbf{v}_{\mathcal{D}_{1} \theta}\left(\Theta_{\mathcal{D}_{2}}\right)=\frac{1}{2} \int\left\{-2 \partial_{x} \theta_{1} \wedge \theta_{2} \wedge \partial_{x} \theta_{2}-2 \partial_{x}^{2} \theta_{2} \wedge \theta_{1} \wedge \theta_{2}\right\} d x \\
=-\int \partial_{x}\left(\theta_{1} \wedge \theta_{2} \wedge \partial_{x} \theta_{2}\right) d x \\
=0,
\end{gathered}
$$

and similarly we have

$$
\operatorname{pr} \mathbf{v}_{\mathcal{D}_{2} \theta}\left(\Theta_{\mathcal{D}_{1}}\right)=0
$$

since

$$
\begin{gathered}
\Theta_{\mathcal{D}_{1}}=\frac{1}{2} \int\left\{-\theta_{1} \wedge \partial_{x} \theta_{2}-\theta_{2} \wedge \partial_{x} \theta_{1}\right\} d x \\
=-\int\left\{\theta_{1} \wedge \partial_{x} \theta_{2}\right\} d x .
\end{gathered}
$$

Therefore the modified Euler-Poisson equation (mEP) is bihamiltonian for $m=1$, and this completes the proof of Theorem 3 .

\section{REFERENCES}

[Ad] R.A. Adams, Sobolev Spaces, Academic Press, Inc. 1975. MR0450957 (56:9247)

[AK] V.I. Arnold and B.A. Khesin, Topological Methods in Hydrodynamics, Springer-Verlag, New York 1998. MR1612569 (99b:58002)

[Arn] V. Arnold, Sur la géométrie différentielle des groupes de Lie de dimension infinie et ses applications à l'hydrodynamique des fluides parfaits, Ann. Inst. Fourier, Grenoble 16 (1966).

[BB] J.P. Bourguignon and H. Brezis, Remarks on the Euler equation, J. Functional Analysis 15 (1974). MR0344713 (49:9452)

[BDP] J.C. Brunelli, A. Das and Z. Popowicz, Deformed Harry Dym and Hunter-Zheng equations, J. Math. Phys. 45 (2004). MR2067579 (2005a:37117)

[BG] M.S. Baouendi and C. Goulaouic, Sharp estimates for analytic pseudodifferential operators and application to Cauchy problems, J. Diff. Eq. 48 (1983). MR696869 (84g:35173) 
[BSS] R. Beals, D. Sattinger and J. Szmigielski, Multi-peakons and a theorem of Stieltjes, Inverse Problems 15 (1999), L1-L4. MR1675325

[Di] J. Dieudonné, Foundations of Modern Analysis, Academic Press, New York and London, 1960. MR0120319 (22:11074)

[Eb1] D.G. Ebin, Espace des métriques riemanniennes et mouvement des fluides via les variétés d'applications, Centre de Mathématiques de l'Ecole Polytechnique et Université Paris 8 1972. MR0464298 (57:4231)

[Ee] J. Eells, A setting for global analysis, Bull. Amer. Math. Soc. 72 (1966). MR0203742 $(34: 3590)$

[EMa] D.G. Ebin and J. Marsden, Groups of diffeomorphisms and the motion of an incompressible fluid, Ann. Math. 92 (1970). MR0271984 (42:6865)

[HM1] A.A. Himonas and G. Misiołek, Remarks on an integrable evolution equation, Geometry and analysis on finite and infinite dimensional Lie groups, Banach Center Publ. 55, Polish Acad. Sci., Warsaw (2002). MR1911981 (2003c:35138)

[HM2] A.A. Himonas and G. Misiołek, The Cauchy problem for an integrable shallow-water equation, Differential Integral Equations 14 (2001). MR1828326 (2002c:35228)

[HM3] A.A. Himonas and G. Misiołek, Analyticity of the Cauchy problem for an integrable evolution equation, Math. Ann. 327 no.3 (2003). MR2021030 (2004m:35232)

[HNS] M. Haragus, D.P. Nicholls, D.H. Sattinger, Solitary Wave Interactions of the EulerPoisson Equations, J. Math. Fluid Mech. 5, (2003). MR1966646 (2004a:76143)

[K] T. Kato, Liapunov functions and monotonicity in the Navier-Stokes equation, Lecture Notes in Math., 1450, Springer, Berlin 1990. MR1084601 (92a:35131)

[KaM] T. Kato and K. Masuda, Nonlinear evolution equations and analyticity I, Ann. de l'Inst. H. Poincare 3, 1986. MR870865 (88h:34041)

[KM] B.A. Khesin and G. Misiołek, Euler equations on homogeneous spaces and Virasoro orbits, Adv. Math. 176, 2003. MR1978343 (2004h:37125)

[LiSat] Y. Li and D.H. Sattinger, Soliton Collisions in the Ion Acoustic Plasma Equations, J. Math. Fluid Mech. 1, (1999). MR1699021

[Mis1] G. Misiołek, Classical solutions of the periodic Camassa-Holm equation, GAFA 12 (2002). MR1937835 (2003k:37125)

[Mis2] G. Misiołek, Stability of flows of ideal fluids and the geometry of the group of diffeomorphisms, Indiana Univ. Math. 42 (1993). MR1218713 (94j:58027)

[Mis3] G. Misiołek, A shallow water equation as a geodesic flow on the Bott-Virasoro group, J. Geom. Phys. 24 (1996). MR1491553 (99d:58018)

[MRW] J.E. Marsden, T. Ratiu, A. Weinstein, Semidirect products and reduction in mechanics, Trans. Amer. Math. Soc. 281 (1984). MR719663 (84k:58081)

[Nir] L. Nirenberg, An abstract form of the nonlinear Cauchy-Kowalevski theorem, J. Differential Geom. 6 (1972). MR0322321 (48:683)

[Nis] T. Nishida, A note on a theorem of Nirenberg, J. Differential Geom. 12 (1977). MR512931 (80a:58013)

[OK] V. Ovsienko and B. Khesin, Korteweg-de Vries superequations as an Euler equation, Functional Anal. Appl. 21 (1987). MR925082 (89g:58095)

[Ol] P.J. Olver, Applications of Lie groups to differential equations, Springer-Verlag, New York (1986). MR836734 (88f:58161)

[Ovs1] L.V. Ovsjannikov, A singular operator in a scale of Banach spaces, Dokl. Akad. Nauk SSSR, 163 (1965). MR0190754 (32:8164)

[Ovs2] L.V. Ovsjannikov, A nonlinear Cauchy problem in a scale of Banach spaces, Dokl. Akad. Nauk SSSR, 200 (1971); Soviet Math. Dokl. 12 (1971). MR0285941 (44:3158)

[S] E.M. Stein, Singular Integrals and Differentiability Properties of Functions, Princeton University Press 1970. MR0290095 (44:7280)

[Sat] D.H. Sattinger, Scaling, mathematical modelling, 8 Integrable systems, DMV-Seminar Series, Band 28, Birkhauser 1998. MR1661770 (2000b:35250)

[Tay1] M.E. Taylor, Pseudodifferential Operators and Nonlinear PDE, Birkhäuser Boston 1991. MR1121019 (92j:35193)

[Tay2] M.E. Taylor, Finite and infinite dimensional Lie groups and evolution equations, Classroom Notes, University of North Carolina-Chapel Hill, Spring 2003.

[Tre] F. Treves, An abstract nonlinear Cauchy-Kovalevska theorem, Trans. Amer. Math. Soc. 150 (1970). MR0274911 (43:669) 
[Tru] E. Trubowitz, The inverse problem for periodic potentials, Comm. Pure Appl. Math. 30, 1977. MR0430403 (55:3408)

[Y] T. Yamanaka, Note on Kowalevskaja's system of partial differential equations, Comment. Math. Univ. St. Paul. 9 (1960). MR0126044 (23:A3341)

Department of Mathematics, University of New Orleans, Lake Front, New Orleans, LOUISIANA 70148 